\title{
The Effects of Meditative Motivation on Mental Health in Those Who Experienced Chakra Meditation: Mediating Effect of Health State
}

\author{
HeeJung Lee ${ }^{1}$ and Sela $\operatorname{Lim}^{2} *$ \\ ${ }^{1}$ Dept. of Naturopathic, Dongbang Culture University, Seongbuk-gu, Seoul, Korea \\ ${ }^{2}$ Dept. of Clinical Psychology, NungIn University, Paltan-myeon, Hwaseong-si, \\ Gyeonggi-do, Korea \\ sera325@nate.com
}

\begin{abstract}
This study explored correlation of People of chakra meditation program operation agency in Seoul of Korea for the chakra meditation experience motivation, and mental health to confirm mediation model and path of physical and emotional and social and subjective health state. To this end, data was collected from 223 survey results conducted by 250. The survey consisted of, the Chakra Meditation Experience Motivation scale, the Mental Health (SCL90R) Questionnaire and The Korean Health Status Measure. The results were analyzed by SPSS macro program. For the research result, this study presented descriptive statics for each variable of respondents' and conducted correlation analysis among chakra meditation experience motivation (Selbstfindung) to mental health (depression) through emotional health state (B=0.886, CI: [0.159-1.857]). However, the path model on the relationship among Chakra meditation experience motivation (Selbstfindung) and emotional health state and mental health(depression) was verified and suggested as well. Afterwards, the author discussed the contribution and limitation of this study, and suggestions for further research.
\end{abstract}

Keywords: Chakra meditation experience motivation, Mental health, State of health, Mediation effect

\section{Introduction}

The chakra meditation experience motivation is conceptualized by Lee [1]. chakra meditation is the self-acceptance in a healthy form. This variable is important because the chakra meditation experience motivation could provide an emotional well-being and physical health and mental stress for human life, helping to see clearly without negative attitude [2]. Therefore, it has a positive effect on the evaluation of the quality of the meditation motivation with the chakra [3]. Heath state leads to and mental health [4]. In addition, the factor structure of the meditation motivation is validated and health state and mental health state of chakra meditation experienced people are being studied [5]. In this regard, it has been reported that, in many studies, compassion, escapism, self-exploration, self-love and emotional stability of chakra meditation experienced people with a positive attitude of mental health is relatively higher than that of chakra meditation experienced people with the positive health state (physical and

Article history:

Received (June 14, 2019), Review Result (August 8, 2019), Accepted (September 12, 2019) 
emotional and social and subjective health state) [2][5][6]. Furthermore, previous studies [2][7][8] report on the effects of the chakra meditation experience motivation on health state in explaining mental health of women and men [9][10]. In the present study, we aimed to study the path model of the chakra meditation experience motivation to mental.

\section{Methods}

\subsection{Subjects and data collection}

This study was conducted from August 2018 to September 2010, for those who participated in the chakra meditation program operation agency in Seoul of Korea. Finally, the data of total 223 participants were analyzed [Table 1].

Table 1. General characteristics of subjects

\begin{tabular}{|c|c|c|c|c|}
\hline & \multirow{2}{*}{ - } & \multicolumn{2}{|c|}{ Subjects } & \multirow{2}{*}{$\%$} \\
\hline & & Male & Female & \\
\hline \multirow{5}{*}{ Ages } & Over the age of 40 & \multicolumn{2}{|c|}{23} & 10.31 \\
\hline & Over the age of 50 & \multicolumn{2}{|c|}{77} & 34.53 \\
\hline & Over the age of 60 & \multicolumn{2}{|c|}{87} & 39.01 \\
\hline & Over the age of 70 & \multicolumn{2}{|c|}{29} & 13.00 \\
\hline & Missing value & \multicolumn{2}{|c|}{7} & 3.14 \\
\hline & Total & \multicolumn{2}{|c|}{223} & 100.00 \\
\hline
\end{tabular}

\subsection{Measures}

\subsubsection{Chakra meditation experience scale}

The questionnaire on meditation methods of subjects who experienced chakra meditation included the following: Meditation motivation scale was developed by Ha and modified by Kim [11][12]. A total of items are composed of 4 sub-factors. Sub-factors consist of compassion, escapism, self-exploration, self-love and emotional stability. The higher the total score, the higher the motivation for meditation. In this study, the total of Cronbach's a was .936.

\subsubsection{Mental health: Symptom checklist090-R (SCL-90-R) scale}

In order to measure mental health, we used the Korean standardized version of Symptom Checklist-90-Revision (SCL-90-R) [13] which was developed by Derogates, Lipman \& Covi. [14] In the present study, the total of the Cronbach's $\alpha$ was .856.

\subsubsection{State of health}

In order to measure physical and emotional and social and subjective health state, we used the State of Health Inventory (K-SHI), which was developed by Shin [15] which was developed by Kim [16] and we used the State of Subjective Health Inventory (K-SSHI), which was developed by Speak, Cowart and Pellet [17] which was developed by Hwang [18]. We refer to the questionnaire which is an analysis of the items and the factor analysis that are appropriate for Korean Chakra Meditation Experienced men and women. In the present study, the total of the Health State of Cronbach's $\alpha$ was .929 and Subjective Health State was .926. 


\subsection{Data analysis}

Data collected for this study were analyzed using the SPSS 22.0 version (IBM Corp., Armonk, NY, USA) as follows.

\section{Results}

\subsection{Path model of Selbstfindung and depression interval}

A state of health analysis was conducted to confirm the effects of chakra meditation experience motivation on mental health, and the results are shown in Table 5. Specifically, the Selbstfindung has a significant effect on the satisfaction of emotional health state $(B=-.147, t=-$ 2.323, $\mathrm{p}<.05)$, In addition, the physical health state and emotional health state was found to have a significant effect on depression $(B=-5.071, t=-2.299, \mathrm{p}<.05)$ and $(B=-6.039, t=-4.047$, $\mathrm{p}<.001)$, and the Selbstfindung was found to have a significant effect depression $(\mathrm{B}=1.905$, $\mathrm{t}=2.186, \mathrm{p}<.05)$. [Table 2].

Table 2. The Analysis of path model of Selbstfindung and depression interval

\begin{tabular}{|c|c|c|c|c|c|c|}
\hline \multicolumn{3}{|c|}{ Path } & \multirow{2}{*}{$\frac{\mathrm{B}}{-0.052}$} & \multirow{2}{*}{$\begin{array}{c}\text { S.E } \\
0.045\end{array}$} & \multicolumn{2}{|c|}{$\mathrm{t}$} \\
\hline \multirow{4}{*}{ Selbstfindung } & $\rightarrow$ & Physical health state & & & -1.161 & - \\
\hline & $\rightarrow$ & Emotional health state & -0.147 & 0.063 & -2.323 & $*$ \\
\hline & $\rightarrow$ & Social health state & -0.067 & 0.062 & -1.076 & - \\
\hline & $\rightarrow$ & \multirow{4}{*}{ Depression } & 1.905 & 0.871 & 2.186 & $*$ \\
\hline Physical health state & $\rightarrow$ & & -5.071 & 2.206 & -2.299 & $*$ \\
\hline Emotional health state & $\rightarrow$ & & -6.039 & 1.492 & -4.047 & $* * *$ \\
\hline Social health state & $\rightarrow$ & & 0.411 & 1.580 & 0.260 & - \\
\hline
\end{tabular}

Table. 3 The mediator effect of Selbstfindung and depression interval

\begin{tabular}{|c|c|c|c|c|c|c|}
\hline \multicolumn{3}{|c|}{ Path } & \multirow{2}{*}{$\begin{array}{c}\text { Total } \\
\text { effect } \\
\mathrm{a} \rightarrow \mathrm{c} \\
\mathrm{a} \rightarrow \mathrm{b} \rightarrow \mathrm{c}\end{array}$} & \multirow[b]{2}{*}{$\begin{array}{l}\text { Direct } \\
\text { effect } \\
a \rightarrow c\end{array}$} & \multirow{2}{*}{$\begin{array}{c}\text { Total } \\
\text { indirect } \\
\text { effect } \\
\mathrm{a} \rightarrow \mathrm{b} \rightarrow \mathrm{c}\end{array}$} & \multirow[b]{2}{*}{$\begin{array}{c}\text { Indirect effect } \\
\text { [bootstrapping } \\
\text { CI 95\%] }\end{array}$} \\
\hline $\begin{array}{c}\text { Independent } \\
\text { variable } \\
\text { A } \\
\end{array}$ & $\begin{array}{c}\text { Parameter } \\
\text { B } \\
\end{array}$ & $\begin{array}{c}\text { Dependent } \\
\text { variable } \\
\text { C } \\
\end{array}$ & & & & \\
\hline \multirow{3}{*}{ Selbstfindung } & Physical health state & \multirow{3}{*}{ Depression } & \multirow{3}{*}{3.029} & \multirow{3}{*}{1.905} & \multirow{3}{*}{1.124} & $\begin{array}{c}0.265 \\
{[-0.211 \sim 0.874]}\end{array}$ \\
\hline & Emotional health state & & & & & $\begin{array}{c}0.886 \\
{[0.159 \sim 1.857]}\end{array}$ \\
\hline & Social health state & & & & & $\begin{array}{c}-0.027 \\
{[-0.335 \sim 0.205]}\end{array}$ \\
\hline
\end{tabular}

The measurement model of Selbstfindung and depression interval. We performed bootstrapping by extracting 2000 parts of the Selbstfindung of chakra meditation experience motivation to the depression of mental health through the emotional health state of health state. As a result, the indirect effect was not significant because the path through the satisfaction of heterosexual satisfaction included 0 at $-.202 \sim .019$ in the $95 \%$ confidence interval of the indirect effect ( $\mathrm{B}=0.886, \mathrm{CI}:$ [0.159 1.857]) [Table 3] [ Figure 1]. 


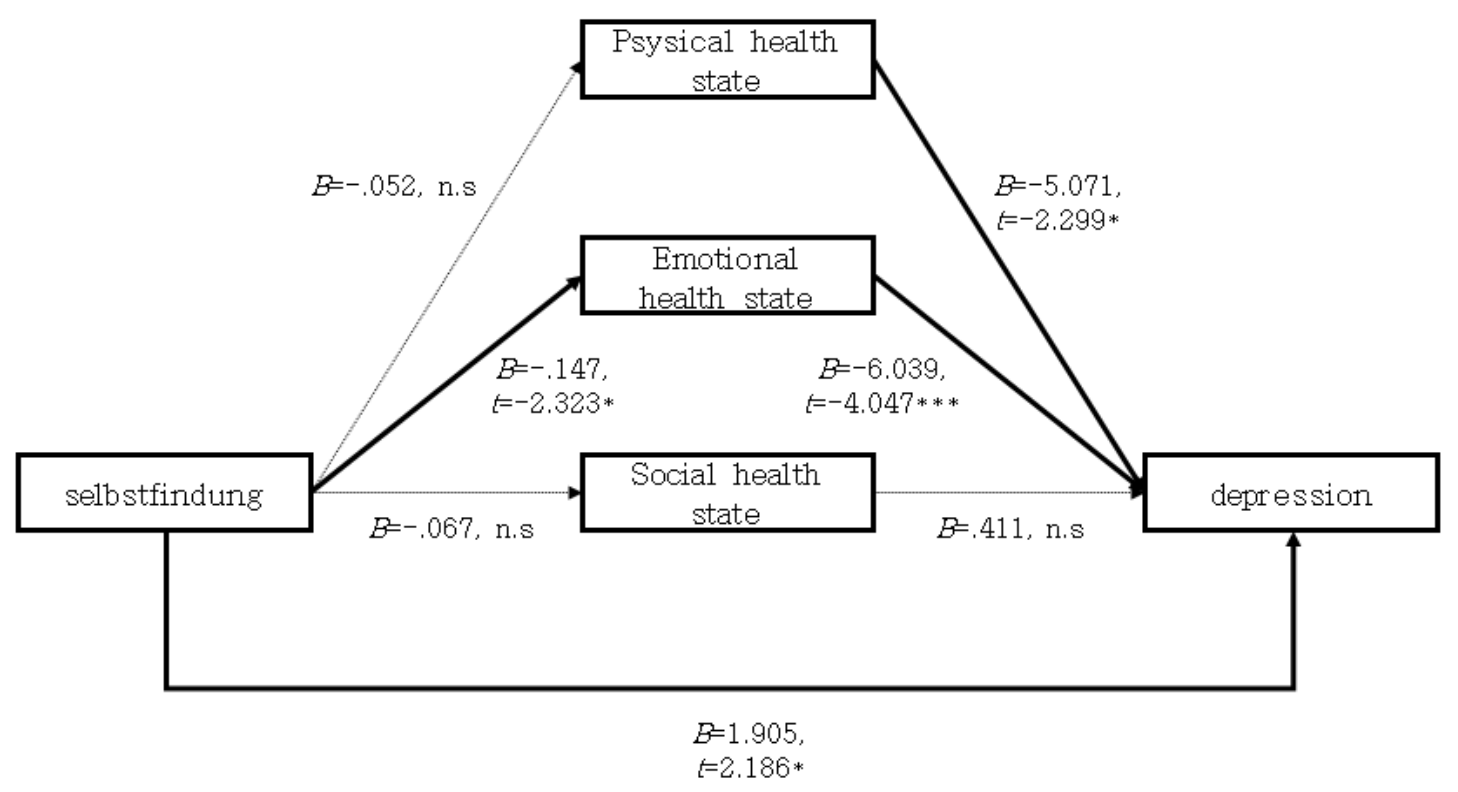

Figure 1. Measurement model of the mediator effect of Selbstfindung and depression interval

\subsection{Path model of escapism and depression interval}

A state of health analysis was conducted to confirm the effects of chakra meditation experience motivation on mental health, and the results are shown in Table 5. Specifically, the escapism has a significant effect on the satisfaction of physical health state $(B=-.095, t=2.148$, $\mathrm{p}<.05)$, In addition, the emotional health $(\mathrm{B}=-.281, \mathrm{t}=-4.690, \mathrm{p}<.001)$ and social health state $(\mathrm{B}=-.178, \mathrm{t}=-2.967, \mathrm{p}<.01)$ was found to have a significant effect on depression and physical health state $(B=-5.919, t=-2.788, p<.01)$ and emotional health state $(B=-4.655, t=-3.168, p<.01)$ was found to have a significant effect on depression. Specifically, the escapism was found to have a significant effect depression $(B=3.825, t=4.420, \mathrm{p}<.001)$ [Table 4].

Table 4. The analysis of path model of escapism and depression interval

\begin{tabular}{|c|c|c|c|c|c|c|}
\hline \multicolumn{3}{|c|}{ Path } & \multirow{2}{*}{$\frac{B}{-0.095}$} & \multirow{2}{*}{$\frac{\text { S.E }}{0.044}$} & \multicolumn{2}{|c|}{$\mathrm{t}$} \\
\hline \multirow{4}{*}{ Escapism } & $\rightarrow$ & physical health state & & & -2.148 & $*$ \\
\hline & $\rightarrow$ & emotional health state & -0.281 & 0.060 & -4.690 & $* * *$ \\
\hline & $\rightarrow$ & social health state & -0.178 & 0.060 & -2.967 & $* *$ \\
\hline & $\rightarrow$ & \multirow{4}{*}{ Depression } & 3.825 & 0.865 & 4.420 & $* * *$ \\
\hline Physical health state & $\rightarrow$ & & -5.919 & 2.123 & -2.788 & $* *$ \\
\hline Emotional health state & $\rightarrow$ & & -4.655 & 1.470 & -3.167 & $* *$ \\
\hline Social health state & $\rightarrow$ & & 0.821 & 1.513 & 0.543 & - \\
\hline
\end{tabular}

About the Measurement model of Escapism and depression interval, we performed bootstrapping by extracting 2000 parts of the escapism of chakra meditation experience motivation to the depression of mental health through the emotional health state of health state. 
As a result, the indirect effect was not significant because the path through the satisfaction of heterosexual satisfaction included 0 at $-.202 \sim .019$ in the $95 \%$ confidence interval of the indirect effect ( $\mathrm{B}=1.306, \mathrm{CI}$ : [0.357 2.627]) [Table 5] [ Figure 2].

Table 5. The mediator effect of escapism and depression interval

\begin{tabular}{|c|c|c|c|c|c|c|}
\hline \multicolumn{3}{|c|}{ Path } & \multirow[b]{2}{*}{$\begin{array}{c}\text { Total effect } \\
\underset{a \rightarrow c}{a \rightarrow b \rightarrow c}\end{array}$} & \multirow[b]{2}{*}{$\begin{array}{c}\text { Direct effect } \\
\qquad a \rightarrow c\end{array}$} & \multirow{2}{*}{$\begin{array}{c}\text { Total } \\
\text { indirect } \\
\text { effect } \\
\mathrm{a} \rightarrow \mathrm{b} \rightarrow \mathrm{c}\end{array}$} & \multirow[b]{2}{*}{$\begin{array}{c}\text { Indirect effect } \\
\text { [bootstrapping CI } \\
95 \%]\end{array}$} \\
\hline $\begin{array}{c}\text { Independent } \\
\text { variable } \\
\text { a } \\
\end{array}$ & $\begin{array}{c}\text { Parameter } \\
\text { B }\end{array}$ & $\begin{array}{c}\text { Dependent } \\
\text { variable } \\
\text { C } \\
\end{array}$ & & & & \\
\hline \multirow{3}{*}{ Escapism } & Physical health state & \multirow{3}{*}{ Depression } & \multirow{3}{*}{5.547} & \multirow{3}{*}{3.825} & \multirow{3}{*}{1.722} & $\begin{array}{c}0.562 \\
{[-0.023 \sim 1.359]}\end{array}$ \\
\hline & Emotional health state & & & & & $\begin{array}{c}1.306 \\
{[0.357 \sim 2.627]}\end{array}$ \\
\hline & Social health state & & & & & $\begin{array}{c}-0.146 \\
{[-0.775 \sim 0.349]}\end{array}$ \\
\hline
\end{tabular}

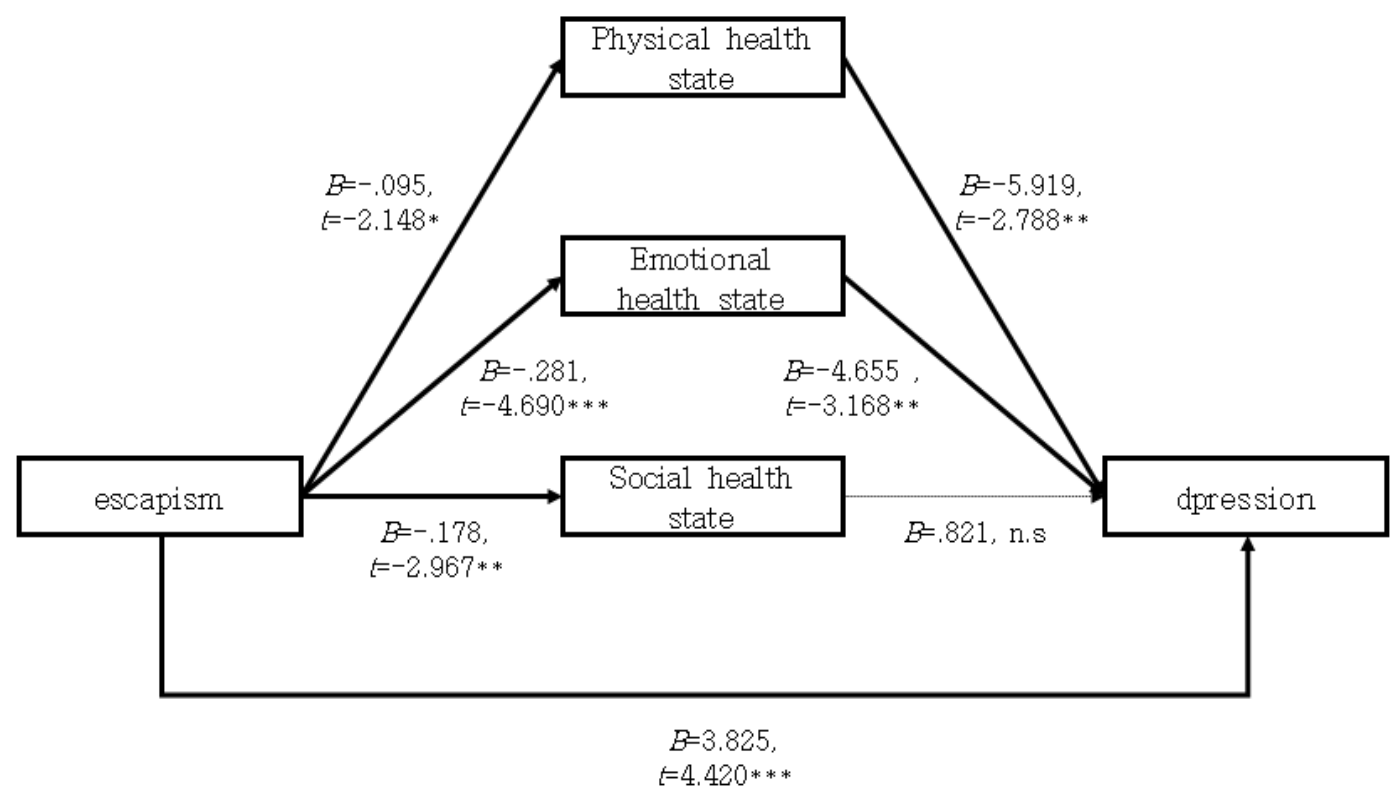

Figure 2. Measurement model of the mediator effect of escapism and depression interval

\section{Conclusions}

The results of this study suggest that Selbstfindung and escapism of chakra meditation experience motivation has a significant effect on the depression of mental health through the emotional health state of health state. Meanwhile, in the simple correlation analysis, it was found that there was a significant correlation between Selbstfindung and escapism and emotional health state and depression, whereas the path search for the median model analysis was not significant. This is consistent with the previous study[2], which reported a significant correlation between Selbstfindung and escapism of chakra meditation experience motivation and 
depression of mental health. Song [2] found in chakra experienced people that most of the causes of Selbstfindung and escapism that interfere with depression after chakra meditation experienced, in daily life. Kim[5] found in chakra activation yoga program experienced people that most of the causes of Selbstfindung and escapism that interfere with thinking are related to the stress symptoms and responses, individual emotional healthy lifestyle and the experiences of depression in daily life. Choi and Seo [19] found in chakra meditation experienced people that most of the causes of Selbstfindung and escapism that interfere with emotional healthy life and depression are related to the tress responses, individual lifestyle, role, and the experiences in daily life. Kabat-Zinn [20] reported that the Meditation is an important factor affecting depression. and Naga [21] reported that was a significant correlation between yoga with chakra meditation and depression. Pilkington, Kirkwood, Rampes and Richardson [22] and Field [23] and Narthaniels [24] reported the research evidence about meditation and depression closely interacts with each other. Therefore, subjective and objective perception of changes in these health conditions could lead to deterioration of psychological function, in conclusion, the mediating effect of chakra meditation experience motivation on mental health and health state in chakra meditation experienced people was solely analyzed in Korea, and significant results were obtained. Nevertheless, it is important to note that this result of the study could be used to refer to mental health when intervening in counseling and clinical setting.

\section{References}

[1] J. S. Lee, "A feast of Zen meditation," Seoul: Nungin Publishing Corp, (2015)

[2] Y. G. Song, "The development and effectiveness of mental health program using Chakra meditation for adults," M.S. thesis, Nungin University, (2017)

[3] S. L. Lim, "The development and effectiveness of mental health program using Chakra meditation for adults," Studies on Buddhist Art and Culture, vol.9, no.9, pp.311-331, (2017)

[4] M. D. Lee, "The effects of the physical and emotional activities the health, self perceived health status and the depression of the elderly," M.S. thesis, School of Nursing Gachon University, (2012)

[5] S. H. Kim, "A study on the development of Chakra activation yoga program and its effects -with a focus on the effect for relieving stress symptoms and response, negative automatic thinking and positive automatic thinking," Ph.D. dissertation, Seoul Buddhist Graduate School University, (2013)

[6] J. S. Kim, "The effects of the second Chakra harmony program on the sexual attitude, personal relationship, and social support," Ph.D. dissertation, Changwon National University, (2009)

[7] E. S. Choi, "The effects of Chakra music on the body, breathing, mind, and consciousness in middle-school students," M.S. thesis, Changwon National University, (2005)

[8] Y. J. Ha, "The affect of Chakra meditation program on happiness of middle-aged women," M.S. thesis, International University of Korea, (2012)

[9] S. S. Doh, "The effects of a Chakra Mantra meditation program on the reduction of depression \& anxiety of middle-aged women," M.S. thesis, Changwon National University, (2011)

[10] N. Y. Kim, "The influence of adult attachment of Korean adults on mental health and health behaviors: mediating role of relationship satisfaction," M.S. thesis, Nungin University, (2018)

[11] K. U. Kim, "A study on the effect of duration and motive for meditation on psychological well-being," M.S. thesis, The Graduate School of Seoul Cyber University, (2016)

[12] H. J. Ha, "Influence of meditator's psychological characteristics on experiences of mindfulness meditation," M.S. thesis, Seoul National University, (2007)

[13] K. I. Kim, J. H. Kim, and H. T. Won, “Korean manual of symptom checklist-90-revision,” Seoul: Choung-Ang Juk-Sung Publishers, (1984) 
[14] L. Derrogatis, R. Lipman, and I. Covi, "The SCL-90: An outpatient psychiatric rating scale," Psychopharmacology Bulletin, (1973)

[15] H. C. Shin, C. H. Kim, B. L. Cho, J. W. Won, S. W. Song, Y. K. Park, Y. H. Yun, and S. P. Chung, "Development of the Korean health status measure," Journal of the Korean Academy of Family Medicine, vol.23, no.4, pp.440-457, (2002)

[16] J. S. Kim, "Comparisons of health status and health care needs between institutionalized and community-living elders," M.S. thesis, Chonbuk University, (2007)

[17] D. L. Speake, M. E. Cowart, and K. Pellet, "Health perception and life styles of the elderly," Research in Nursing \& Health, vol.12, pp.93-100, (1989) DOI: 10.1002/nur.4770120206

[18] M. H. Hwang, “A structural model for self-care of elderly and psychological well-being,” Ph.D. dissertation, Kyungpook national university, (2002)

[19] J. Y. Choi and K. H. Seo, "The effects of yoga practice on stress responses," Korean Journal of Clinical Psychology, vol.10, no.4, pp.455-473, (2005)

[20] J. Kabat-Zinn, "Mindfulness-based interventions in context: Past, present, and future," Clinical Psychology: Science and Practice, vol.10, no.2, pp.144-156, (2003) DOI: 10.1093/clipsy.bpg016

[21] K. Naga, "Yoga: A spiritual practice with therapeutic value in psychiatry," Asian Journal of Psychiatry, vol.5, no.2, pp.186-189, (2012) DOI: 10.1016/j.ajp.2012.05.003

[22] K. Pilkington, G. Kirkwood, H. Rampes, and J. Richardson, "Yoga for depression: the research evidence," Journal of Affective Disorders, vol.89, no.1, pp.13-24, (2005) DOI: 10.1016/j.jad.2005.08.013

[23] T. Field, "Yoga clinical research review," Complementary the Rapies in Clinical Practice, vol.17, no.1, pp.1-8, (2011) DOI: 10.1016/j.ctcp.2010.09.007

[24] L. Nathaniel's, "Yoga for all," Nursing Times, Jan, pp.52-54, (1984) 
The Effects of Meditative Motivation on Mental Health in Those Who Experienced Chakra Meditation: Mediating Effect of Health State

This page is empty by intention. 